\title{
ESCALAS DE VALORACIÓN DE DOLOR EN PACIENTES CRÍTICOS NO COMUNICATIVOS: REVISIÓN SISTEMÁTICA
}

\author{
PAIN VALUATION SCALES IN NON-COMMUNICABLE CRITICAL PATIENTS: \\ SYSTEMATIC REVIEW \\ ESCALAS DE VALORAÇÃO DE DOR EM PACIENTES CRÍTICOS NÃO \\ COMUNICATIVOS:REVISÃO SISTÉMICA
}

\section{Edith Rivas Riveros}

edith.rivas@ufrontera.cl

Universidad de La Frontera. Temuco. Chile

ORCID: 0000-9832-4534

Macarena Alarcón Pincheira

Universidad de La Frontera. Temuco. Chile

ORCID: 0000-0003-4769-0881

\section{Valeria Gatica Cartes}

Universidad de La Frontera. Temuco. Chile

ORCID: 0000-0003-1777-6801

\section{Karina Neupayante Leiva}

Universidad de La Frontera. Temuco. Chile

ORCID: 0000-0002-3808-3695

\section{$\mathbf{M}^{\mathbf{a}}$ Belén Schneider Valenzuela}

Universidad de La Frontera. Temuco. Chile

ORCID: 0000-0001-9059-2270

DOI: https://doi.org/10.22235/ech.v7i1.1544

Enviado: 06/02/2018

Aceptado: $15 / 03 / 2018$

Resumen: La valoración del dolor se percibe con dificultad cuando los pacientes se encuentran en estado crítico, ya que su condición imposibilita el autoinforme. El objetivo fue determinar fiabilidad de escalas de valoración del dolor en paciente crítico no comunicativo (escalas BPS, CPOT, NVPS, ESCID y Campbell). Se trató de una revisión sistemática en bases de datos electrónicas: Pubmed, ScienceDirect, BVS, Scielo y Biblioteca Cochrane; consultando a las revistas electrónicas Nursing in Critical Care, Journal of Pain and Symptom Management, Pain Medicine, Pain Management Nursing, Critical Care, Acta Anaesthesiologica Scandinavica, Enfermería Intensiva, Intensive Care Medicine, International Journal of Nursing Studies, Archives of Trauma Research, Revista Brasileña de Terapia Intensiva y The Journal of Pain. Los criterios de elegibilidad aplicados: pacientes críticos con incapacidad para comunicarse de manera verbal, escrita y/o a través de gesticulaciones intencionales; mayores de 18 años; de ambos sexos; que las 
escalas contengan la propiedad psicométrica alfa de Cronbach que mide tanto confiabilidad (consistencia interna) como validez de criterio; que los artículos se encuentren en su versión completa; en idioma inglés, portugués y español y actualizados, con menos de diez años. La evaluación de los estudios y resumen de resultados, se centró en: a) Base de Datos/ Revista, Muestra, Diseño, Escala y $\alpha$ de Cronbach. b) Calidad de los estudios incluidos, c) Niveles de evidencia Scottish Intercollegiate Guidelines Network y de calidad de la evidencia científica; asimismo se ha valorado Riesgo de sesgo. De 114 registros, 13 artículos fueron incluidos en la revisión para finalmente seleccionar 4 estudios de escala Behavioral Pain Scale que alcanzó la mayor fiabilidad en un estudio realizado en Estados Unidos en el cual el Alfa de Cronbach fluctúa entre 0,80 a 0,92 . La BPS demostró tener mayor confiabilidad y validez de criterio para valorar el dolor en los pacientes en estudio. La fortaleza de los resultados es la magnitud y relevancia del problema que otorga fundamento a la necesidad de continuar desarrollando evidencia de alta calidad (estudios de mayor rigor metodológico) para responder a las necesidades de los pacientes. Asimismo, los resultados son componentes esenciales para la toma de decisiones, que deben verse reflejadas en Guía Clínica de manejo de pacientes no comunicativos. Se concluye que es necesario continuar fomentando el desarrollo de investigaciones en esta línea, que evalúan un tema tan sensible como el dolor, el cual el Ministerio de Salud lo ha catalogado como el quinto signo vital.

Palabras Clave: Cuidados Críticos, Dolor, Dimensión del Dolor, Umbral del Dolor, Manejo del Dolor.

Abstract: The assessment of pain is perceived with difficulty when patients are in critical condition, since their condition makes self-report impossible. The objective was to determine the reliability of pain assessment scales in critical non-communicative patients (BPS, CPOT, NVPS, ESCID and Campbell scales). It was a systematic review in electronic databases: Pubmed, ScienceDirect, VHL, Scielo and Cochrane Library; consulting the electronic journals Nursing in Critical Care, Journal of Pain and Symptom Management, Pain Medicine, Pain Management Nursing, Critical Care, Acta Anaesthesiologica Scandinavica, Intensive Nursing, Intensive Care Medicine, International Journal of Nursing Studies, Archives of Trauma Research, Journal Brazilian Institute of Intensive Therapy and The Journal of Pain. Eligibility criteria applied: critical patients with inability to communicate verbally, in writing and / or through intentional gestures; over 18 years; of both sexes; that the scales contain Cronbach's alpha psychometric property that measures both reliability (internal consistency) and criterion validity; that the articles are in their full version; in English, Portuguese and Spanish and updated, with less than ten years. The evaluation of the studies and summary of results, focused on: a) Database / Magazine, Sample, Design, Scale and Cronbach's $\alpha$. b) Quality of included studies, c) Scottish Intercollegiate Guidelines Network evidence levels and quality of scientific evidence; Likewise, risk of bias has been assessed. Of 114 records, 13 articles were included in the review to finally select 4 studies of scale Behavioral Pain Scale that reached the highest reliability in a study conducted in the United States in which the Cronbach's Alpha fluctuated between 0.80 to 0.92 . BPS showed greater reliability and criterion validity to assess pain in the patients under study. The strength of the results is the magnitude and 
relevance of the problem that gives rise to the need to continue developing high quality evidence (studies of greater methodological rigor) to respond to the needs of patients. Likewise, the results are essential components for decision-making, which should be reflected in the Clinical Guide for non-communicative patient management. It is concluded that it is necessary to continue fostering the development of research in this line, which evaluates a subject as sensitive as pain, which the Ministry of Health has cataloged as the fifth vital sign.

Keywords: Critical Care, Pain, Dimension of Pain, Pain threshold, Pain management.

Resumo: A avaliação da dor é percebida com dificuldade quando os pacientes estão em estado crítico, uma vez que sua condição torna o autorrelato impossível. O objetivo foi determinar a confiabilidade das escalas de avaliação da dor em pacientes críticos não comunicativos (escalas BPS, CPOT, NVPS, ESCID e Campbell). Revisão sistemática nas bases de dados eletrônicas: Pubmed, ScienceDirect, BVS, Scielo e Cochrane Library; Consulta de enfermagem revistas eletrônicas em Critical Care, Journal of Pain e Gestão Sintoma, Medicina da Dor, Enfermagem Pain Management, Critical Care, Acta anaesthesiologica Scandinavica, Enfermagem Intensiva, Medicina Intensiva, Jornal Internacional de Estudos de Enfermagem, Archives of Trauma Research, Journal Instituto Brasileiro de Terapia Intensiva e The Journal of Pain. Critérios de elegibilidade aplicados: pacientes críticos com incapacidade de se comunicar verbalmente, por escrito e / ou por meio de gestos intencionais; mais de 18 anos de idade; de ambos os sexos; que as escalas contêm a propriedade psicométrica alfa de Cronbach que mede tanto a confiabilidade (consistência interna) quanto a validade de critério; que os artigos estão em sua versão completa; em inglês, português e espanhol e atualizado, com menos de dez anos. A avaliação dos estudos e resumo dos resultados, teve como foco: a) Banco de Dados / Revista, Amostra, Design, Escala e $\alpha$ de Cronbach. b) Qualidade dos estudos incluídos, c) Diretrizes Intercolegiadas Escocesas Evidências da rede e qualidade das evidências científicas; Da mesma forma, o risco de viés foi avaliado. 114 registos, 13 itens foram incluídos na avaliação para seleccionar finalmente 4 estudos escala comportamental Escala de Dor que atingiram a mais elevada fiabilidade num estudo realizado nos EUA, em que a alfa de Cronbach variando 0,80-0,92. BPS mostrou maior confiabilidade e validade de critério para avaliar a dor nos pacientes em estudo. A força dos resultados é a magnitude e importância do problema que dá substância à necessidade de continuar a desenvolver evidência de alta qualidade (estudos de maior rigor metodológico) para atender as necessidades dos pacientes. Da mesma forma, os resultados são componentes essenciais para a tomada de decisões, que devem ser refletidos no Guia Clínico para o gerenciamento não comunicativo do paciente. Conclui-se que é necessário continuar a promover o desenvolvimento de pesquisas nesta linha, avaliando uma questão tão sensível como a dor, que o Ministério da Saúde tem listado como o quinto sinal vital.

Palavras-chave: Cuidados Críticos. Dor Dimensão da dor. Limiar de dor. Gestão da dor 


\section{INTRODUCCIÓN}

El dolor, según la Asociación Internacional del Dolor (IASP), es definido como "una experiencia sensorial subjetiva y emocional desagradable, asociada con una lesión presente o potencial" (1). Esta experiencia, al ser subjetiva, varía entre individuos y es influenciada por creencias y emociones del paciente, orígenes y significados personales, siendo aceptado por algunos y rechazado por otros, por lo que la forma más fiable de cuantificarlo es mediante la manifestación verbal del paciente $(2,3)$. No obstante, existen pacientes cuyo estado de salud no les permite comunicarse verbalmente, tal como pacientes hospitalizados en Unidades Críticas que además pueden estar intubados o bajo sedación. Esto dificulta la medición del dolor, siendo necesario recurrir a la observación de otros indicadores o conductas.

Para este estudio se considerará crítico a "un paciente hemodinámicamente inestable, que requiere de cuidados, drogas vasoactivas, ventilación mecánica, monitorización continua y que no puede expresar en forma verbal, escrita o con lenguaje corporal la intensidad de su dolor" (4). Según Gélinas, los indicadores fisiológicos, es decir los signos vitales, son inespecíficos para evaluar el dolor en este tipo de pacientes, ya que pueden variar de acuerdo al estado y diagnóstico, por lo que deben ser utilizados con cautela (5). Esto es respaldado por un estudio realizado por Young, en el que los parámetros hemodinámicos aumentaron independientemente de si el procedimiento realizado era nociceptivo o no (6). Es por esto que los indicadores del comportamiento son los recomendados para este fin, al representar información válida en estos pacientes $(7,8)$. Existen diversas escalas para evaluar este tipo de pacientes basadas en indicadores de comportamiento: Escala Conductual del Dolor (BPS), Escala Observacional del Dolor en Cuidados Críticos (CPOT), Escala de Campbell, Escala Sobre Conductas Indicadoras de Dolor (ESCID) y Escala de Dolor en el adulto No Comunicativo (NVPS) (9, 10). Sin embargo, en Chile se carece de estudios para validar este tipo de instrumentos. Esto constituye un problema clínico debido a que estudios ratifican que el uso de estos instrumentos produce disminución en la administración de analgésicos y sedantes, siendo útil en la evaluación de la efectividad de intervenciones farmacológicas y contribuyendo a que ésta sea oportuna y adecuada para cada paciente de manera individualizada (11).

Bajo esta perspectiva la calidad de la atención se incluye dentro de las metas sanitarias del Ministerio de Salud, Gobierno de Chile, correspondientes al periodo 2011-2020 (12). Esto cobra mayor relevancia en el contexto de las Unidades de Cuidados Intensivos (UCI), en donde la toma de decisiones debe ser rigurosa debido a la condición inestable y crítica de los pacientes. El dolor es un evento frecuente en estos servicios, con una incidencia de hasta $50 \%$, y su manejo debe ser considerado un aspecto trascendental, especialmente en pacientes con dificultad para comunicarse, ya que su tratamiento inefectivo puede generar complicaciones que agravan su estado de salud (13). Esto se debe a que las manifestaciones fisiológicas son variadas, como taquicardia, taquipnea, palidez o rubicundez de la piel, sudoración, hipertensión, midriasis, ansiedad o depresión, inquietud psicomotora y/o insomnio (14). Según Claret, el dolor no tratado produce diversas complicaciones a nivel de sistemas, como alteraciones cardiovasculares, gastrointestinales y renales (1). Además reduce la movilidad del paciente, lo que puede provocar trombosis venosa profunda, 
embolia pulmonar y neumonía. A la luz de lo anteriormente descrito es imprescindible brindar tratamiento oportuno y adecuado para disminuir el riesgo de complicaciones, lo que conlleva a una disminución de la estadía en UCI y brindar atención de calidad (15).

Según el Ministerio de Salud una atención sanitaria de calidad "es aquella que identifica las necesidades de salud de los individuos o de la población de una forma total y precisa, que destina los recursos necesarios (humanos y otros) a estas necesidades de forma oportuna y tan efectiva como el estado actual del conocimiento lo permita". Llevado al marco legal, Chile presenta un modelo de garantías en salud que incorpora la garantía de calidad. A partir de esto el decreto con fuerza de ley $\mathrm{N}^{\circ} 1$ de 2005 establece los estándares mínimos que deberán cumplir los prestadores institucionales de salud, tales como hospitales, clínicas, consultorios, centros médicos, con el objetivo de garantizar que las prestaciones alcancen la calidad requerida para la seguridad de los usuarios (16). Esto, ligado al Código Deontológico del CIE para la profesión de enfermería, relacionado a la práctica, "establecer normas de atención de salud y un contexto de trabajo que fomente la seguridad y la calidad de los cuidados", tiene directa relación con el tema de estudio ya que radica en el cumplimiento del rol profesional. El ser gestores del cuidado implica la detección correcta de las necesidades del paciente y las intervenciones adecuadas para mejorar su calidad de vida (17). Tiene además la responsabilidad profesional, ética y legal para controlar el dolor y aliviar el sufrimiento. El tratamiento del dolor en la UCI requiere conocimiento sobre el impacto del dolor en el bienestar del paciente, así como del compromiso profesional para el adecuado control del dolor (2).

El objetivo general fue determinar la mejor fiabilidad en escalas de valoración del dolor en paciente crítico no comunicativo (BPS, CPOT, NVPS, ESCID y Campbell). El objetivo específico fue recopilar la evidencia disponible por medio de estrategias de búsqueda reproducible y en diferentes fuentes.

\section{METODOLOGÍA}

Se trató de una revisión sistemática, para responder la pregunta de investigación: ¿Cuál de las escalas de valoración del dolor en paciente crítico no comunicativo (BPS, CPOT, NVPS, ESCID y Campbell) presenta mejor fiabilidad? Para la estrategia de búsqueda y recolección de datos. Dos revisores independientes (la investigadora principal y un tercero con formación en búsqueda bibliográfica) identificaron en el título y resumen los artículos con información del tema tratado, de acuerdo a los criterios de elegibilidad previamente definidos. Los artículos fueron clasificados en las categorías: "incluido", "excluido" y "dudoso". Los dudosos fueron leídos en su metodología para reclasificarlos como incluidos o excluidos. Luego, los revisores abrieron sus clasificaciones y se estudió el grado de acuerdo. En caso de no acuerdo respecto a algún artículo evaluado se resolvió por consenso entre ambos revisores.

En cuanto a la localización y selección de estudios, se utilizaron las bases de datos electrónicas: Pubmed, ScienceDirect, CINHAL, BVS, Scielo, Biblioteca Cochrane y ISI Web of Science. Adicionalmente se consultaron las revistas electrónicas: Nursing in Critical Care, Journal of Pain and Symptom Management, Pain Medicine, Pain Management Nursing, Critical Care, Acta Anaesthesiologica Scandinavica, Enfermería 
Intensiva, Intensive Care Medicine, International Journal of Nursing Studies, Archives of Trauma Research, Revista Brasileña de Terapia Intensiva y The Journal of Pain. Se utilizaron descriptores controlados extraídos del Medical Subjects Headings (MeSH). También se utilizaron palabras no controladas extraídas de las palabras claves de los artículos seleccionados y que abordan todos los términos de la pregunta PICoR.

Se realizaron dos búsquedas, la primera para identificar el número de escalas de valoración de dolor en paciente crítico no comunicativo disponibles y posteriormente una búsqueda por cada escala para determinar su fiabilidad. La investigación expone los resultados de estudios primarios en relación al análisis del coeficiente Alfa de Cronbach de las escalas de evaluación del dolor en pacientes críticos no comunicativos (18).

Para la extracción de datos, se trabajó con plantillas diseñadas para el estudio. Se consideraron tanto estudios principales como artículos relacionados. En la primera búsqueda se utilizaron los términos "Pain scale and uncommunicative critical patient"; se seleccionaron inicialmente todos los estudios que tenían relación con la pregunta de investigación. Cuando el título era poco claro, se utilizaron los abstract y finalmente el artículo integral para evaluar la pertinencia del estudio. Se identificaron un total de 35 artículos, y estas escalas: Escala Conductual del Dolor (BPS), Escala Observacional del Dolor en Cuidados Críticos (CPOT), Escala de Dolor en el adulto No Comunicativo (NVPS), Escala de Campbell y Escala Sobre Conductas Indicadoras de Dolor (ESCID). La segunda búsqueda se centró en las propiedades psicométricas de confiabilidad y validez de cada escala. Se utilizaron los términos "Behavioral Pain Scale" para la Escala Conductual del Dolor (BPS), para la escala Escala Observacional del Dolor en Cuidados Críticos (CPOT), se utilizaron los términos "Critical-Care Pain Observation Tool", luego para la Escala de Dolor en el Adulto No Comunicativo (NVPS) los términos utilizados fueron "Pain Scale in Non-Communicative Adult"; finalmente para la Escala Sobre Conductas Indicadoras de Dolor (ESCID) se utilizaron los términos "Scale of Behavior Indicators of Pain”.

En tabla 1 se muestran resultados de búsquedas de acuerdo a las bases de datos y combinaciones de descriptores.

Tabla N¹: Búsquedas de acuerdo a las bases de datos y combinaciones de descriptores.

\begin{tabular}{l|c|c|c|c|c|c}
\hline Combinación Descriptores & $\begin{array}{c}\text { ESCI } \\
D\end{array}$ & BPS & NVPS & CPOT & $\begin{array}{l}\text { ESCI } \\
D\end{array}$ & Total \\
Bases de datos & 25 & 10 & 29 & 3 & 1 & 68 \\
\hline Pubmed & 9 & 10 & 3 & 0 & 0 & 22 \\
\hline ScienceDirect & 1 & 6 & 16 & 0 & 0 & 23 \\
\hline BVS & 0 & 1 & 0 & 0 & 0 & 1 \\
\hline Scielo & 0 & 0 & 0 & 0 & 0 & 0 \\
\hline Biblioteca Cochrane & 35 & 27 & 48 & 3 & 1 & 114 \\
\hline Total & & & & & & \\
\hline
\end{tabular}

Fuente: Elaboración Propia (2017) 
La totalidad de artículos encontrados son internacionales, siendo los principales países Canadá, Brasil, EEUU y China. A partir de estos resultados, se seleccionaron los estudios que cumplían con los siguientes criterios de inclusión:

- Aplicados en pacientes críticos con incapacidad para comunicarse de manera verbal, escrita y/o a través de gesticulaciones intencionales.

- Que contengan la propiedad psicométrica alfa de Cronbach que mide tanto confiabilidad (consistencia interna) como validez de criterio.

- Que se encuentren en su versión completa.

- Realizados en mayores de 18 años.

- Realizados en pacientes de ambos sexos.

- En idioma inglés, portugués y español.

- Actualizados con menos de 10 años.

La valoración de la validez de los estudios identificados en la búsqueda, se centró en: Base de Datos/ Revista, Muestra, Diseño, Escala y $\alpha$ de Cronbach. En cuanto a los posibles sesgos, cabe destacar que existen estudios en los que se analizan las propiedades psicométricas de más de una escala y que por lo tanto se repiten. Es por esta razón que el total de artículos seleccionados corresponden a 13.

El método de consistencia interna basado en el alfa de Cronbach permite estimar la fiabilidad de un instrumento de medida a través de un conjunto de ítems que se espera que midan el mismo constructo o dimensión teórica. La fiabilidad de la consistencia interna del instrumento se puede estimar con el alfa de Cronbach. La medida de la fiabilidad mediante el alfa de Cronbach asume que los ítems (medidos en escala tipo Likert) miden un mismo constructo y que están altamente correlacionados (19). Cuanto más cerca se encuentre el valor del alfa a 1 mayor es la consistencia interna de los ítems analizados. La fiabilidad de la escala debe obtenerse siempre con los datos de cada muestra para garantizar la medida fiable del constructo en la muestra concreta de investigación. Como criterio general, George y Mallery sugieren las recomendaciones siguientes para evaluar los coeficientes de alfa de Cronbach: -Coeficiente alfa $>.9$ es excelente - Coeficiente alfa $>.8$ es bueno -Coeficiente alfa $>.7$ es aceptable - Coeficiente alfa $>.6$ es cuestionable - Coeficiente alfa $>.5$ es pobre $(20)$.

Tomado en cuenta el riesgo de sesgo, se aplicó a cada estudio un listado de estímulos que permitió evaluar el potencial riesgo de sesgo, evaluándose muestra (muestras pequeñas), aleatorización, grupo control tipo de diseño, inconsistencia de los resultados, imprecisión (referida a si los intervalos de confianza son amplios). De los 13 seleccionados, 4 estudios no mencionan el tipo de diseño; todas están publicadas en revistas indexadas ScienceDirect con buen factor de impacto. En muestra, existe variabilidad en su potencia, (rango de 15 a 100 pacientes incluidos); en 8 estudios se menciona pacientes con ventilación mecánica, sedados e inconscientes y en 5 describe pacientes. 
Tabla N²: Características de los artículos seleccionados.

\begin{tabular}{|c|c|c|c|c|c|}
\hline Publicación, Año y lugar & $\begin{array}{l}\text { Base de Datos/ } \\
\text { Revista }\end{array}$ & Muestra & Diseño & Escala & $\begin{array}{l}\alpha \text { de } \\
\text { Cronbach }\end{array}$ \\
\hline $\begin{array}{l}\text { Cultural adaptation and } \\
\text { psychometric properties of the } \\
\text { Portuguese version of the scale } \\
\text { Behavioral Pain Scale - intubated } \\
\text { patients (BPS-IP / PT). } 2013 \text {. } \\
\text { Coimbra }{ }^{21} \text {. }\end{array}$ & $\begin{array}{l}\text { Scielo/ } \\
\text { Enfermagem Referencia } \\
\text { FI: } 1.082\end{array}$ & $\begin{array}{l}60 \text { pacientes con } \\
\text { ventilación mecánica e } \\
\text { incapaz de autoinforme } \\
\text { de dolor }\end{array}$ & $\begin{array}{l}\text { Observacional con } \\
\text { traducción y } \\
\text { análisis de validez }\end{array}$ & BPS & $0,65-0,73$ \\
\hline $\begin{array}{l}\text { Validation of the Brazilian version } \\
\text { of Behavioral Pain Scale in adult } \\
\text { sedated and mechanically ventilated } \\
\text { patients. } 2015 \text {. Brasil } 22 \text {. }\end{array}$ & $\begin{array}{l}\text { ScienceDirect/ The } \\
\text { Journal of pain } \\
\text { FI: } 4.463\end{array}$ & $\begin{array}{l}35 \text { pacientes adultos } \\
\text { sedados o inconscientes } \\
\text { con ventilación mecánica }\end{array}$ & Transversal & BPS & 0,80 \\
\hline $\begin{array}{l}\text { Use of a Behavioural Pain Scale to } \\
\text { assess pain in ventilated, } \\
\text { unconscious and/or } \\
\text { sedated patients. } 2006 \text {. Australia }{ }^{6} \text {. }\end{array}$ & $\begin{array}{l}\text { Science Direct/ } \\
\text { Enfermeria de Cuidados } \\
\text { Intensivos y Criticos FI: } \\
0.462\end{array}$ & 44 pacientes & Prospectivo & BPS & 0,64 \\
\hline $\begin{array}{l}\text { Reliability and validity of the } \\
\text { Chinese version of the behavioral } \\
\text { pain scale in intubated and non- } \\
\text { intubated critically ill patients: Two } \\
\text { cross-sectional studies. } 2016 \text {. } \\
\text { Beijing }{ }^{23} \text {. }\end{array}$ & $\begin{array}{l}\text { Pubmed, ScienceDirect/ } \\
\text { International Journal of } \\
\text { Nursing Studies } \\
\text { FI: } 3.561\end{array}$ & $\begin{array}{l}\text { Estudio } 1: 129 \text { pacientes } \\
\text { ( } 53 \text { intubados y } 76 \text { no } \\
\text { intubados). Estudio } 2: 83 \\
\text { ( } 43 \text { intubados y } 40 \text { no } \\
\text { intubados }\end{array}$ & Transversal & BPS & $\begin{array}{l}0,724 \text { a } \\
0,743\end{array}$ \\
\hline $\begin{array}{l}\text { Validity and sensitivity of } 6 \text { pain } \\
\text { scales in critically ill, intubated } \\
\text { adults. } 2015 \text {. EstadosUnidos }{ }^{24} \text {. }\end{array}$ & $\begin{array}{l}\text { Pubmed/ American } \\
\text { Journal of Critical Care. } \\
\text { FI: } 1.870\end{array}$ & $\begin{array}{l}150 \text { pacientes de UCI } 50 \\
\text { comunicativos y } 100 \text { no } \\
\text { comunicativos con } \\
\text { ventilación mecánica }\end{array}$ & Observación & BPS & 0,80 a 0,92 . \\
\hline $\begin{array}{l}\text { Psychometric Analysis of } \\
\text { Behavioral Pain Scale Brazilian } \\
\text { Version in Sedated and } \\
\text { Mechanically Ventilated Adult } \\
\text { Patients: A Preliminary Study }{ }^{25} \text {. } \\
\text { 2015. Brasil }\end{array}$ & $\begin{array}{l}\text { Portal Regional de la } \\
\text { BVS/Pain Practice } \\
\text { FI: } 2.317\end{array}$ & $\begin{array}{l}15 \text { pacientes } \\
\text { neurológicos, sedados y } \\
\text { con ventilación mecánica. }\end{array}$ & Transversal & BPS & $\begin{array}{l}\text { R: } 0,42 \\
\text { LO: } 0,53 \\
\text { AE: } 0,57\end{array}$ \\
\hline $\begin{array}{l}\text { Translation and cultural adaptation } \\
\text { of the Brazilian Portuguese version } \\
\text { of the Behavioral Pain Scale. } 2014 \text {. } \\
\text { Brasil }{ }^{26} \text {. }\end{array}$ & $\begin{array}{l}\text { Scielo/Revista } \\
\text { Brasileña de Terapia } \\
\text { Intensiva } \\
\text { FI: } 0.47\end{array}$ & $\begin{array}{l}100 \text { pacientes intubados, } \\
\text { ventilados } \\
\text { mecánicamente, } y \\
\text { sedados }\end{array}$ & No Especifica & BPS & De 0,501. \\
\hline $\begin{array}{l}\text { Validation of the Scale of Behavior } \\
\text { Indicators of Pain (ESCID) in } \\
\text { critically ill, non-communicative } \\
\text { patient sunder mechanical } \\
\text { ventilation: results of the ESCID } \\
\text { scale.2011. Madrid, España }{ }^{9} \text {. }\end{array}$ & $\begin{array}{l}\text { ScienceDirect, Pubmed/ } \\
\text { Enfermeria Intensiva } \\
\text { FI: } 0.462\end{array}$ & 42 pacientes & $\begin{array}{l}\text { Observacional } \\
\text { desarrollo y } \\
\text { validación de una } \\
\text { escala }\end{array}$ & ESCID & $\begin{array}{l}\text { Osciló entre } \\
0,70-0,80\end{array}$ \\
\hline $\begin{array}{l}\text { Comparison of two pain assessment } \\
\text { tools in non verbal critical care } \\
\text { patients. } 2011 \text {. Estados Unidos }{ }^{27} \text {. }\end{array}$ & $\begin{array}{l}\text { ScienceDirect/ Pain } \\
\text { Management Nursing } \\
\text { FI: } 1.745\end{array}$ & 100 pacientes & $\begin{array}{l}\text { Descriptivo, } \\
\text { Comparativo, } \\
\text { Prospectivo } \\
\end{array}$ & CPOT & 0,76 \\
\hline $\begin{array}{l}\text { A preliminary validation of the } \\
\text { Swedish version of the critical-care } \\
\text { pain observation tool in adults. } \\
\text { 2011. Estocolmo, Suecia }{ }^{28} \text {. }\end{array}$ & $\begin{array}{l}\text { BVS/Acta } \\
\text { Anaesthesiologica } \\
\text { Scandinavica FI: } 2.049\end{array}$ & $\begin{array}{l}40 \text { pacientes conscientes e } \\
\text { inconscientes }\end{array}$ & No Especifica & CPOT & $\begin{array}{l}\text { Desde } 0,31 \\
\text { hasta } 0,81\end{array}$ \\
\hline $\begin{array}{l}\text { Pain Assessment Tool in the } \\
\text { Critically ill Post-Open Heart } \\
\text { Surgery Patient Population. } 2010 . \\
\text { New Jersey }{ }^{29} \text {. }\end{array}$ & $\begin{array}{l}\text { ScienceDirect/ Pain } \\
\text { Management Nursing } \\
\text { FI: } 1.745\end{array}$ & 24 pacientes & $\begin{array}{l}\text { Prospectivo de } \\
\text { cohortes }\end{array}$ & $\begin{array}{l}\text { CPOT y } \\
\text { BPS }\end{array}$ & $\begin{array}{l}\text { BPS: } 0,70 \\
\text { CPOT: } 0,71\end{array}$ \\
\hline $\begin{array}{l}\text { Psychometric comparison of three } \\
\text { behavioural scales for the } \\
\text { assessment of pain in critically ill } \\
\text { patients unable to self-report.2014. } \\
\text { Chicago }{ }^{30} \text {. }\end{array}$ & $\begin{array}{l}\text { Pubmed, BVS/ Critical } \\
\text { Care } \\
\text { FI: } 4.950\end{array}$ & 30 pacientes & No Especifica & $\begin{array}{l}\text { CPOT, } \\
\text { BPS y } \\
\text { NVPS }\end{array}$ & $\begin{array}{l}\text { BPS: } 0,80 \\
\text { CPOT: } 0,81 \\
\text { NVPS: } 0,76\end{array}$ \\
\hline $\begin{array}{l}\text { Pain measurement in mechanically } \\
\text { ventilated critically ill patients: } \\
\text { Behavioral Pain Scale versus } \\
\text { Critical-Care Pain Observation } \\
\text { Tool. } 2015 \text {. Amsterdam, Holanda }{ }^{31} \text {. }\end{array}$ & $\begin{array}{l}\text { ScienceDirect, BVS/ } \\
\text { Critical Care } \\
\text { FI: } 4.950\end{array}$ & $\begin{array}{l}68 \text { pacientes con } \\
\text { ventilación mecánica. }\end{array}$ & $\begin{array}{l}\text { Prospectivo de } \\
\text { cohortes }\end{array}$ & $\begin{array}{l}\text { BPS y } \\
\text { CPOT }\end{array}$ & $\begin{array}{l}\text { BPS: } 0,70 \\
\text { CPOT: } 0,71\end{array}$ \\
\hline
\end{tabular}


Se ha valorado adecuadamente la validez de los estudios seleccionados, Las poblaciones son similares, en todos se recoge la valoración de Alfa de Cronbach. Sobre la calidad de los estudios incluidos, sean de forma independiente o por vía de consenso, los revisores evaluaron independientemente la calidad de cada artículo seleccionado. Y, con respecto a las discrepancias en la valoración de la calidad metodológica, los evaluadores consensuaron un acuerdo; en aquellas donde no hubo acuerdo, un experto actuó como árbitro para decidir la calidad metodológica del artículo en cuestión.

Niveles de calidad de la evidencia cientifica (AATM)

De acuerdo a escala Agència d'Avaluació de Tecnologia Mèdica (AATM), la clasificación tiene en cuenta, además del diseño de los estudios, una valoración específica de su calidad. En este caso de estudios, observacionales y prospectivos, corresponde en calidad: VI (Regular), Estudios de cohorte Multicéntrico Apareamiento; VII (Regular) Estudios de casos y controles Multicéntrico Calidad del estudio y VIII (Pobre) Estudios descriptivos.

\section{Niveles de evidencia Scottish Intercollegiate Guidelines Network (SIGN)}

De acuerdo a la escala (SIGN), incorpora en valor (2+) a estudios de cohortes o de casos y controles bien realizados, con bajo riesgo de confusión, sesgos o azar y una moderada probabilidad de que la relación sea causal. En la investigación los sujetos de estudios corresponden a estudios realizados previamente y que se encuentran disponibles de manera pública en la red; por lo tanto está exento de las regulaciones federales llevadas a cabo por el comité ético de investigación clínica (CEIC), y cabe destacar que en estos estudios queda protegida la privacidad de los pacientes, de tal manera que es imposible conocer la identidad de éstos (21). No se utilizó el consentimiento informado dado que se realizó un análisis secundario de datos, sin contar con pacientes que actúen como participantes. Sin embargo se tuvo presente el rigor metodológico. Esto se respalda a través de la Common Rule y la ley de portabilidad y responsabilidad de seguro sanitario (HIPPA) que autoriza esto siempre y cuando los datos no contengan identificadores específicos de los participantes (22).

\section{RESULTADOS}

Inicialmente se identificaron 114 artículos, para seleccionar 13 artículos (11,4\%), perteneciendo a artículos actualizados donde más del $84 \%$ han sido publicados en los últimos 5 años. En una primera etapa se compararon cuatro escalas (BPS, ESCID, CPOT, NVPS), que evalúan el dolor en este tipo de pacientes. Los resultados se exponen en Tabla 2 . 
Tabla N³: Escalas BPS, ESCID , CPOT, NVPS y $\alpha$ de Cronbach

\begin{tabular}{|c|c|c|}
\hline Nombre escala & Escala & $\alpha$ de Cronbach \\
\hline Scale Behavioral Pain Scale (BPS). 2013. Coimbra (22) & BPS & $0,65-0,73$ \\
\hline Behavioral Pain Scale 2015. Brasil (23) & BPS & 0,80 \\
\hline Behavioural Pain Scale 2006. Australia (6) & BPS & 0,64 \\
\hline Behavioral Pain scale. 2016. Beijing (24) & BPS & 0,724 a 0,743 \\
\hline Behavioral Pain scale 2015. EstadosUnidos (25) & BPS & 0,80 a 0,92 . \\
\hline Behavioral Pain Scale 2015. Brasil (26) & BPS & \begin{tabular}{|l|} 
R: 0,42 \\
LO: 0,53 \\
AE: 0,57 \\
\end{tabular} \\
\hline Behavioral Pain Scale. 2014. Brasil (27) & BPS & De 0,501 . \\
\hline $\begin{array}{l}\text { Behavior Indicators of Pain (ESCID) 2011. Madrid, } \\
\text { España (9) }\end{array}$ & $\begin{array}{l}\text { ESCI } \\
\mathrm{D}\end{array}$ & \begin{tabular}{|l|} 
Osciló entre \\
$0,70-0,80$ \\
\end{tabular} \\
\hline Critical care patients.2011. Estados Unidos (28) & CPOT & 0,76 . \\
\hline $\begin{array}{l}\text { Critical-care pain observation. 2011. Estocolmo, Suecia } \\
\text { (29) }\end{array}$ & CPOT & $\begin{array}{l}\text { Desde } 0,31 \text { hasta } \\
0,81 \text {. }\end{array}$ \\
\hline $\begin{array}{l}\text { Pain Assessment Tool in the Critically ill Post-Open } \\
\text { Heart Surgery Patient Population. 2010. New Jersey } \\
\text { (30) }\end{array}$ & $\begin{array}{l}\text { CPOT } \\
\text { y BPS }\end{array}$ & $\begin{array}{l}\text { BPS: } 0,70 \\
\text { CPOT: } 0,71\end{array}$ \\
\hline $\begin{array}{l}\text { Comparison of three behavioural scales for the } \\
\text { assessment of pain in critically ill patients unable to } \\
\text { self-report.2014. Chicago (31) }\end{array}$ & $\begin{array}{l}\text { CPOT, } \\
\text { BPS y } \\
\text { NVPS }\end{array}$ & \begin{tabular}{|l|} 
BPS: 0,80 \\
CPOT: 0,81 \\
NVPS: 0,76 .
\end{tabular} \\
\hline $\begin{array}{l}\text { Pain measurement in mechanically ventilated criticall } \\
\text { ill patients: Behavioral Pain Scale versus Critical-Care } \\
\text { Pain Observation Tool.2015. Ámsterdam, Holanda (32) }\end{array}$ & $\begin{array}{l}\text { BPS y } \\
\text { CPOT }\end{array}$ & $\begin{array}{l}\text { BPS: } 0,70 \\
\text { CPOT: } 0,71\end{array}$ \\
\hline
\end{tabular}

Fuente: Elaboración Propia (2017)

En una segunda etapa se clasificó la escala BPS de acuerdo a fiabilidad; el principal hallazgo es un estudio realizado en Estados Unidos que evaluó el dolor durante cuatro fases: antes de la exploración física, durante el examen físico, antes de la aspiración endotraqueal y finalmente durante la aspiración endotraqueal. El artículo está en la base Pubmed, es del año 2015, publicado en revista con factor de impacto 1.870, menciona el diseño como observacional, y presenta potencia en muestra y grupo control. (150 pacientes de UCI 50 comunicativos y 100 no comunicativos con ventilación mecánica). Presentó un coeficiente de Alfa de Cronbach que va de 0,80 a 0,92, el cual otorga, entre los artículos seleccionados, la mayor confiabilidad y validez de criterio a través de este estadígrafo a la Escala conductual del dolor (BPS).

Tabla N4 Escala Behavioral Pain Scale y índice de fiabilidad 


\begin{tabular}{l|l|l}
\hline Nombre escala & Escala & $\boldsymbol{\alpha}$ de Cronbach \\
\hline Behavioral Pain Scale 2015. Brasil (23) & BPS & 0,80 \\
\hline Behavioral Pain scale. 2016. Beijing (24) & BPS & 0,724 a 0,743 \\
\hline Behavioral Pain scale 2015. Estados Unidos (25) & BPS & 0,80 a 0,92. \\
\hline $\begin{array}{l}\text { Comparison of three behavioural scales for the assessment } \\
\text { of pain in critically ill patients unable to self-report.2014. } \\
\text { Chicago (31) }\end{array}$ & BPS & BPS: 0,80 \\
\hline
\end{tabular}

Fuente: Elaboración Propia (2017)

Con la finalidad de marcar un precedente que permita a futuro estandarizar la atención en relación a la utilización de estos instrumentos y como fin último aumentar la calidad de vida de los usuarios, se plantea la necesidad de realizar investigaciones en la línea y validación de escalas respectivas.

\section{DISCUSIÓN}

Al ser el dolor un evento frecuente en los pacientes hospitalizados se transforma en un aspecto fundamental, que se dificulta en aquellos que no pueden manifestarlo de forma verbal. Sin embargo, en nuestro país, pese a la existencia de escalas a nivel mundial para realizar esta labor fundamental, las cuales son basadas en comportamientos y respuestas fisiológicas independientes de la verbalización del paciente, esta medición no se realiza, quedando completamente subjetiva de acuerdo a la percepción del profesional de enfermería, cambiando significativamente según el criterio de cada uno de ellos.

El objetivo de este estudio es identificar qué escala presenta mayor fiabilidad, lo cual se realizó mediante una revisión sistemática en diversas bases de datos, llegando a un resultado en el cual se identificó que todas las escalas son válidas y confiables según el estadígrafo Alfa de Cronbach. Tras esto nace la inquietud de saber porqué no se implementa una de estas escalas en Chile, preferentemente la de mayor Alfa de Cronbach, para realizar una valoración objetiva y disminuir la cifra actual de dolor en los pacientes críticos no comunicativos, correspondiente al $50 \%$ de los ingresos de la Unidad de Cuidados Intensivos. Una de las fortalezas de los resultados es la magnitud y relevancia del problema que otorga fundamento a la necesidad de continuar desarrollando evidencia de alta calidad, (estudios de mayor rigor metodológico), para responder a las necesidades de los pacientes. Asimismo, los resultados, son componentes esenciales para la toma de decisiones, que debe verse reflejada en Guía Clínica de manejo de pacientes no comunicativos.

Las limitaciones fueron en la búsqueda, donde se encontraron artículos protegidos con derechos de autor, y no se pudo obtener los estudios en versión completa. Por lo tanto quedaron descartados mediante los criterios de exclusión e inclusión, limitando la selección. Conjuntamente, se identificó un estudio en idioma coreano, lo que limitó su traducción. 


\section{CONCLUSIONES}

La BPS demostró ser la escala con mayor confiabilidad y validez de criterio para valorar el dolor en pacientes críticos no comunicativos, categorizada de buena a excelente según el coeficiente Alfa de Cronbach. Es necesario continuar fomentando el desarrollo de investigaciones en esta línea, que evalúan un tema tan sensible como el dolor, el cual el Ministerio de Salud lo ha catalogado como el quinto signo vital, tema que resalta la importancia de su valoración y contribuye a reforzar la práctica ética de los profesionales del cuidado de la salud, orientada a la aplicación de instrumentos confiables y validados que permitan evaluar el dolor, especialmente en pacientes con problemas en la comunicación (33). Se agrega a ello el entorno de ambiente hospitalario y la condición del paciente, lo cual agrava el dolor experimentado por presencia de ansiedad, temor, y molestia del mismo usuario o de sus familiares; por ello es imprescindible evaluar la condición con la mejor evidencia disponible.

\section{REFERENCIAS BIBLIOGRÁFICAS}

1. Claret M. Escalas de evaluación de dolor y protocolo de analgesia en terapia intensiva. [Internet]. Argentina: Instituto Argentino de Diagnóstico y Tratamiento; 2012 [Citado 22 Ago de 2016]; Pág. 5-6. Disponible en: http://www.sati.org.ar/files/kinesio/monos/MONOGRAFIA\%20Dolor\%20-\%20Cla rett.pdf

2. Pagliuco T, Marinilza L, Matheus R. Valoración de la experiencia del dolor postoperatorio en pacientes en unidades de cuidados intensivos. Rev Bras Ter Intensiva [Internet] 2011 [Citado 22 de Ago de 2016]; 24(4): 5-6. Disponible en: http://www.scielo.br/scielo.php?script=sci_arttext\&pid=S0103-507X201100040001 2\&lang=es

3. Vázquez M, Pardavila M, Maldonado M, Lizaldre Y, Coscojuela M, Asiain M. Evaluation of pain during posture change in patients with invasive mechanical ventilation. Enferm Intensiva [Internet] 2009 [Citado 22 de Ago de 2016]; 20(1):2-9. Disponible

en: http://www.elsevier.es/es-revista-enfermeria-intensiva-142-articulo-valoracion-deldolor-durante-el-13135724

4. Vargas R. Cuidado humanizado al paciente críticamente enfermo, enfermería pieza clave en la atención. Cien y Cuid [Internet] 2007 [Citado 22 de Ago de 2016]; 4(4): 21-27. Disponible en: https://dialnet.unirioja.es/servlet/articulo?codigo $=2534029$

5. Arbour C, Gélinas C. Are vital signs valid indicators for the assessment of pain in postoperative cardiac surgery ICU adults? Intensive Crit Care Nurs [Internet] 2010 [Citado 22 de Ago de 2016; 20(1): 9-10 Disponible en: https://www.ncbi.nlm.nih.gov/pubmed/20044256

6. Young J, Siffleet J, Nikoletti S, Shaw T. Use of a Behavioral pain scale to assess pain in ventilated, unconscious and/or sedated patients. Intensive Crit Care Nurs [Internet] 2006 [Citado 22 de Ago de 2016]; 22(1): 32-39. Disponible en: http://ezproxy.ufro.cl:2052/science/article/pii/S0964339705000613

7. Gélinas C, Arbour C. Behavioral and physiologic indicators during a nociceptive procedure in conscious and unconscious mechanically ventilated adults: Similar or 
different? J Crit Care [Internet] 2009 [Citado 22 de Ago de 2016]; 24(4): 1-11. Disponible en: https://www.ncbi.nlm.nih.gov/pubmed/19327961

8. Gélinas C, Johnston C. Pain assessment in the critically ill ventilated adult: validation of the Critical-Care Pain Observation Tool and physiologic indicators. Clin J Pain [Internet] 2007 [Citado 22 de Ago de 2016]; 23(6): 497-505. Disponible en: https://www.ncbi.nlm.nih.gov/pubmed/17575489

9. Latorre I, Solís M, Falero T, Larrasquitu A, Romay A, Millán I, et al. Validation of the Scale of Behavior Indicators of Pain (ESCID) in critically ill, non-communicative patients under mechanical ventilation: results of the ESCID scale. Enferm Intensiva [Internet] 2011 [Citado 22 de Ago de 2016]; 22(1): 1-10. Disponible en: http://www.elsevier.es/es-revista-enfermeria-intensiva-142-articulo-validacion-escal a-conductas-indicadoras-dolor-S1130239910001069

10. Fernández L. Valoración de las escalas de dolor en pacientes con ventilación mecánica en Unidad de Cuidados Intensivos [Internet]. España: Universidad de Coruña; 2013 [Citado 22 de Ago de 2016]; Pág. 1-25. Disponible en: http://ruc.udc.es/dspace/bitstream/handle/2183/13611/FernandezRamos_Lorena_TF G_2014.pdf?sequence $=2$

11. Gélinas C, Arbour C, Michaud C, Vaillant F, Desjardins S. Implementation of the critical-care pain observation tool on pain assessment/management nursing practices in an intensive care unit with nonverbal critically ill adults: a before and after study. Int J Nurs Stud [Internet] 2011 [Citado 22 de Ago de 2016]; 48(12): 1-10. Disponible en: https://www.ncbi.nlm.nih.gov/pubmed/21550048

12. Ministerio de Salud Gobierno de Chile. Estrategia Nacional de Salud Para el cumplimiento de los Objetivos Sanitarios de la Década 2011- 2020 [Internet]. Santiago de Chile 2011. [Citado 22 de Ago de 2016]. Pág. 301-317. Disponible en: http://www.minsal.cl/portal/url/item/c4034eddbc96ca6de0400101640159b8.pdf

13. Chanques G, Jaber S, Barbotte E, Violet S, Sebbane M, Perrigault P, et al. Impact of systematic evaluation of pain and agitation in an intensive care unit. Crit Care Med [Internet] 2006 [Citado 22 de Ago de 2016]; 34(6): 9. Disponible en: https://www.ncbi.nlm.nih.gov/pubmed/16625136

14. Goic A. Semiología Médica. 3ra Edición. España: Mediterráneo; 2010.

15. Skrobik Y, Ahern S, Leblanc M, Marquis F, Awissi D, Kavanagh B. Protocolized intensive care unit management of analgesia, sedation, and delirium improves analgesia and subsyndromal delirium rates. Anesth Analg [Internet] 2010 [Citado 22 de Ago de 2016]; 111(2): 169 Disponible en: https://www.ncbi.nlm.nih.gov/pubmed/20375300\%20

16. Velásquez M. Ministerio de Salud Gobierno de Chile. Acreditación de Calidad en Salud [Internet] Santiago de Chile 2011. [Citado 22 de Ago de 2016. Disponible en: http://web.minsal.cl/portal/url/item/b644fa9a8af8d5cce040010164011221.pdf

17. Consejo Internacional de Enfermeras. Código deontológico del CIE para la profesión de Enfermería [Internet] Suiza: Ginebra: 2006. [Citado 22 de Ago de 2016].

Disponible

en: http://www.usal.edu.ar/archivos/medi/otros/14_codigo_deontologico.pdf 
18. Hernández R. Fernández C. Baptista P. Metodología de la Investigación. 5a ed. Perú: McGraw Hill; 2010; 150-151

19. Frías D. Apuntes de SPSS, Análisis de fiabilidad de las puntuaciones de un instrumento de medida. Alfa de Cronbach: un coeficiente de fiabilidad. [Internet] Universidad de Valencia; 2014. [Citado 01 Nov de 2017] Disponible en: https://www.uv.es/friasnav/ApuntesSPSS.pdf

20. George D, Mallery P. SPSS for Windows Step by Step. [Internet] 2003. [Citado 01 Nov de 2017] Disponible en: https://wps.ablongman.com/wps/media/objects/385/394732/george4answers.pdf

21. Hulley S, Cummings S, Browner W, Grady D, Newman T. Diseño de Investigaciones Clínicas. 4aed. Philadelphia: Wolters Kluwer Health; 2014. Pág. 211-259.

22. Cunha L, Figueiredo A, Marques M, Bizarro V. Cultural adaptation and psychometric properties of the Portuguese version of the Behavioral Pain Scale Intubated Patient (BPS-IP/PT). Rev Enf Ref [Internet] 2013 [Citado 22 Ago 2016]; 3(9): 1-10. Disponible en: http://www.scielo.gpeari.mctes.pt/scielo.php?script=sci_arttext\&pid=S0874-028320 $13000100001 \&$ lang $=\mathrm{pt}$

23. Azevedo I, González I, Cerqueira M, Badaue D, Santana V, Melo J. Validation of the Brazilian version of Behavioral Pain Scale in adult sedated and mechanically ventilated patients. Braz J Anesthesiol [Internet] 2016 [Citado 22 Ago 2016]; 11(03): 1-7. Disponible en: http://www.sciencedirect.com/science/article/pii/S0104001416300380

24. Chen J, Lu Q, Ying X, Zhong Y, Chun Y, Yan H. Reliability and validity of the Chinese version of the behavioral pain scale in intubated and non-intubated critically ill patients: Two cross-sectional studies. Int J Nurs Stud [Internet] 2016 [Citado 22 Ago 2016]; 61(1): 63-71. Disponible en: https://www.clinicalkey.com/nursing/\#!/content/playContent/1-s2.0-S00207489163 00621?returnurl=http:\%2F\%2Flinkinghub.elsevier.com $\% 2$ Fretrieve $\% 2 F$ pii $\% 2 \mathrm{FS} 00$ 20748916300621\%3Fshowall\%3Dtrue\&referrer $=\mathrm{http}: \% 2 \mathrm{~F} \% 2 \mathrm{Fwww}$.sciencedirect. com\%2Fscience\%2Farticle\%2Fpii\%2FS0020748916300621

25. Mamoona A, Gan M, Ferguson P, Sherman S, Elswick R. Validity and Sensitivity of 6 Pain Scales in Critically Ill, Intubated Adults. Am J Crit Care [Internet] 2015 [Citado 01 Oct 2016]; 24(6): 514-523. Disponible en: http://ajcc.aacnjournals.org/content/24/6/514.long

26. Azevedo I, Alves I, Badaue D, Santana V, Santana J. Psychometric Analysis of Behavioral Pain Scale Brazilian Version in Sedated and Mechanically Ventilated Adult Patients: A Preliminary Study. Pain Pract [Internet] 2016 [Citado 22 Ago 2016], 16(4): 8. Disponible en: https:/www.ncbi.nlm.nih.gov/pubmed/25857484

27. Morete M, Camargo S, Alves C, Silva A, Odierna M. Translation and cultural adaptation of the Brazilian Portuguese version of the Behavioral Pain Scale. Rev Bras Ter Intensiva [Internet] 2014 [Citado 22 Ago de 2016]; 26(4): 373-378. Disponible en: https:/www.ncbi.nlm.nih.gov/pmc/articles/PMC4304465/

28. Paulson M, Leske J, Maidl C, Hanson A, Dziadulewics L. Comparison of Two Pain Assessment Tools in Nonverbal Critical Care Patients. Pain Manag Nurs [Internet] 
2011 [Citado 22 Ago 2016]; 12(4): 218-224. Disponible en: https://www.ncbi.nlm.nih.gov/pubmed/22117753

29. Nürnberg D, Saboonchi F, Sackey P, Bjorling G. A preliminary validation of the Swedish version of the critical-care pain observation tool in adults. Acta Anaesthesiol Scand [Internet] 2011 [Citado 22 Ago 2016]; 55(4): 375 - 506. Disponible en: http://onlinelibrary.wiley.com/doi/10.1111/j.1399-6576.2010.02376.x/full

30. Marmo L, Fowler S. Pain assessment tool in the critically ill post-open heart surgery patient population. Pain Manag Nurs [Internet] 2010 [Citado 22 Ago 2016], 11(3): 134-140 Disponible en: https://www.ncbi.nlm.nih.gov/pubmed/20728062

31. Chanques G, Pohlman A, Kress J, Molinari N, Jong A, Jaber S, et al. Psychometric comparison of three behavioural scales for the assessment of pain in critically ill patients unable to self-report. Crit Care [Internet] 2014 [Citado 22 Ago 2016]; 18(5): 1-12. Disponible en: https://www.ncbi.nlm.nih.gov/pmc/articles/PMC4220092/pdf/13054_2014_Article_ 2900.pdf

32. Rijkenberg S, Stilma W, Endeman H, Bosman RJ, Oudemans HM. Pain measurement in mechanically ventilated critically ill patients: Behavioral Pin Scale versus Critical - Care Pain Observation Tool. J Crit Care [Internet] 2015 [Citado 22 Ago 2016]; 30(1): 167-172. Disponible en: http://ezproxy.ufro.cl:2052/science/article/pii/S0883944114003852

33. Ministerio de Salud de Chile. MINSAL. Circular Ministerial 2a/46: Evaluación del Dolor como Quinto Signo Vital. 2004. 